ESAIM: M2AN

M2AN, Vol. 41, No 1, 2007, pp. 147-167

DOI: $10.1051 / \mathrm{m} 2 \mathrm{an}: 2007009$
ESAIM: Mathematical Modelling and Numerical Analysis

www.edpsciences.org/m2an

\title{
GENERALIZED COMBINED FIELD INTEGRAL EQUATIONS FOR THE ITERATIVE SOLUTION OF THE THREE-DIMENSIONAL HELMHOLTZ EQUATION
}

\author{
Xavier Antoine ${ }^{1,2}$ And Marion Darbas ${ }^{3}$
}

\begin{abstract}
This paper addresses the derivation of new second-kind Fredholm combined field integral equations for the Krylov iterative solution of tridimensional acoustic scattering problems by a smooth closed surface. These integral equations need the introduction of suitable tangential square-root operators to regularize the formulations. Existence and uniqueness occur for these formulations. They can be interpreted as generalizations of the well-known Brakhage-Werner [A. Brakhage and P. Werner, Arch. Math. 16 (1965) 325-329] and Combined Field Integral Equations (CFIE) [R.F. Harrington and J.R. Mautz, Arch. Elektron. Übertragungstech (AË̈) 32 (1978) 157-164]. Finally, some numerical experiments are performed to test their efficiency.
\end{abstract}

Mathematics Subject Classification. 76Q05, 78A45, 47G30, 35C15, 65F10.

Received: January 9, 2006 .

\section{INTRODUCTION}

Integral equations are widely used in modern acoustic scattering codes for solving large scale problems in the high-frequency regime [26]. These developments have been strongly influenced by the introduction of the the Fast Multipole Method (FMM) of Rokhlin [26,48] and others recent high-order solvers [12,13]. For example, the FMM leads to a computational cost of a matrix-vector product of the order of $\mathcal{O}(n \log n)$ instead of $\mathcal{O}\left(n^{2}\right)$ for a dense complex matrix of size $n$. Generally, a preconditioner is associated to this resolution to get a good convergence rate of the iterative solver and to obtain lower computational times. Numerous works have been devoted to this problem. Maybe the most widely developed approach is based on algebraic solvers like for instance the SPAI preconditioners [18, 19, 23,24], the methods based on the operators splitting [3, 21] or the wavelet-based preconditioners $[22,33]$. The ill-conditioning of the linear system to solve is often linked to the fact that the underlying integral equation is a first-kind Fredholm equation but also to the physics through the coupling of non-local modes $[8,25,26]$. This is for instance the case of the Brakhage-Werner integral

\footnotetext{
Keywords and phrases. Acoustic scattering, Helmholtz equation, second-kind Fredholm integral equation, Krylov iterative solution.

${ }^{1}$ Institut Élie Cartan de Nancy, Université Henri Poincaré Nancy 1, Bureau 307, BP 239, 54506 Vandoeuvre-lès-Nancy, France. Xavier.Antoine@iecn.u-nancy.fr

2 Institut National Polytechnique de Lorraine, École Nationale Supérieure des Mines de Nancy, Département de Génie Industriel, Bureau 495, Parc de Saurupt, CS 14 234, 54042 Nancy Cedex, France. Xavier.Antoine@mines.inpl-nancy.fr

3 Ceremade, Université Paris Dauphine, Place du Maréchal de Lattre de Tassigny, 75775 Paris Cedex 16, France.

darbas@ceremade.dauphine.fr
}

(C) EDP Sciences, SMAI 2007 
formulations [11,29,40] and the Combined Field Integral Equation (CFIE) of Harrington and Mautz [34] for a Neumann boundary condition. We can also cite the Electric Field Integral Equation (EFIE) [26]. Second-kind integral equations have generally a much better convergence rate when they are solved e.g. by the GMRES $[49,50]$. Superlinear convergence phenomenon then occurs $[17,39,47]$. The scattering problem is not elliptic and leads to highly indefinite linear systems of equations. Therefore, algebraic solvers designed for this class of operators should not naturally yield a priori good convergence properties for solving integral equations in diffraction. This suggests that the physical nature of the problems and the mathematical structure of the underlying Helmholtz equation should be more appropriately taken into account in the construction of an efficient preconditioner. Such a tentative has been recently developed by Christiansen and Nédélec [27,28] who have used the Caldèron relations [51] for designing an analytical preconditioner for the EFIE for the scattering problem by an open surface. Another possibility based on the construction of a local preconditioner has also been presented in [8]. However for a closed surface, the EFIE suffers from the presence of interior resonant modes [29]. For these reasons, modified integral formulations as Brakhage-Werner and combined field integral equations, which ensure the existence and the uniqueness of the solution at any frequency [26,29], are generally preferred.

The aim of this paper is to propose some well-conditioned second-kind Fredholm combined field integral equations well-adapted for the iterative solution by a Krylov solver of the three-dimensional acoustic scattering problem by a smooth closed surface. These new formulations can be seen as a generalization of both the Brakhage-Werner and Combined Field Integral Equations. Their construction is based on the integration of efficient pseudodifferential operators to regularize the trace of the single-layer potential or the normal derivative trace of the double-layer potential according to the scattering problem to solve. These regularizing operators expressed as square-root tangential operators are derived from ideas arising in Beam Propagation Methods $[35,44,52]$. They also fall into the general framework of On-Surface Radiation Conditions introduced in the middle of the eighties by Kriegsmann et al. [41] and studied by several authors [4, 5, 9, 16,36-38]. The proposed integral equations are uniquely solvable at any frequency for a closed surface. This is an important property for the applications for instance in inverse scattering problems [30]. These new alternative integral equations are constructed as second-kind Fredholm integral equations and are therefore characterized by an excellent eigenvalue clustering. This central property leads to a superlinear convergence of the GMRES $[17,39,47]$. For the sake of completeness, let us precise that others CFIEs based on different techniques have been recently proposed in $[15,42]$. In [15], Buffa and Hiptmair have constructed, in the case of non-smooth surfaces, a coercive CFIE by regularizing the double-layer operator (instead of the single-layer potential in our case) (see also [14] for the extension to electromagnetism). Since their CFIE is a compact perturbation of the first-kind EFIE, the convergence of the iterative solvers will however still depend on the mesh refinement. No numerical simulations are provided by these authors. Another new well-posed CFIE, proposed by Levadoux and Michielsen [42, 43] and much closer to our approach, has been derived using pseudodifferential operator calculus. The chosen coupling operator between the EFIE and MFIE is introduced as an approximation of the admittance operator (in fact the Dirichlet-to-Neumann map) associated with the scattering problem. For the construction of this operator, the authors have used a partition of unity associated to the boundary of the scatterer. The principle has been extended to electromagnetism [1] and these formulations have been numerically validated to show their efficiency.

The plan of the paper is the following. In Section 2, we present the general problem setting and some basic results about the integral equation representations for the Helmholtz equation. In Section 3, we define the general process for designing some generalizations of the Brakhage-Werner integral equation based on the use of the Dirichlet-Neumann (DN) and Neumann-Dirichlet (ND) maps. This construction starts from the ideas developed in [6]. In Section 4, we construct an efficient and simple approximation of both the DN and ND operators by applying some similar techniques as in the Beam Propagation Methods [35,44] working in a generalized coordinates system. Section 5 is devoted to the well-posedness of the new integral equations at any frequency. In Section 6, we calculate the eigenvalues of the new operators and numerically show that the new integral equations have an excellent eigenvalue clustering even for high-frequencies for the scattering problem of a plane wave by a sphere. In Section 7, we extend the ideas to a generalization of the usual CFIE of Harrington 
and Mautz [34]. We develop several aspects linked to the implementation of the new integral equations in a Krylov iterative solver in Section 8. An essential aspect is that the approximations of the DN and ND operators are computed by a paraxialization technique [46]. In Section 9, we perform some numerical experiments to show that the generalized integral formulations have some interesting convergence rates.

\section{INTEGRAL REPRESENTATIONS FOR ACOUSTIC SCATTERING}

Let us consider a smooth closed bounded set $\Omega^{-} \subset \mathbb{R}^{3}$ whose boundary $\Gamma=\partial \Omega^{-}$is a two-dimensional regular compact manifold. Let us define $\Omega^{+}=\mathbb{R}^{d} \backslash \overline{\Omega^{-}}$as the associated exterior domain of propagation. We consider an incident time-harmonic acoustic wave $u^{\text {inc }}$ defined by the wavenumber $k=2 \pi / \lambda$, setting $\lambda$ as the wavelength of the incident field. The time-dependence is $\mathrm{e}^{-i \omega t}$, denoting by $\omega$ the frequency of the signal. Then, the diffraction boundary value problem can be written as

$$
\left\{\begin{array}{l}
\text { Find } u^{+} \in H_{\mathrm{loc}}^{1}\left(\overline{\Omega^{+}}\right) \text {such that } \\
\Delta u^{+}+k^{2} u^{+}=0, \quad \text { in } \mathcal{D}^{\prime}\left(\Omega^{+}\right), \\
\gamma_{j}^{+} u^{+}=g, \text { in } H^{1 / 2-j}(\Gamma), \quad \text { for } j=0 \text { or } 1, \\
\lim _{|x| \rightarrow+\infty}|x|^{1 / 2}\left(\nabla u^{+} \cdot \frac{x}{|x|}-i k u^{+}\right)=0
\end{array}\right.
$$

where $H_{\text {loc }}^{1}\left(\overline{\Omega^{+}}\right)$designates the Fréchet space

$$
H_{\mathrm{loc}}^{1}\left(\overline{\Omega^{+}}\right):=\left\{v \in \mathcal{D}^{\prime}\left(\Omega^{+}\right) / \psi v \in H^{1}\left(\Omega^{+}\right), \quad \forall \psi \in \mathcal{D}\left(\mathbb{R}^{3}\right)\right\}
$$

The first equation of system (1) is the so-called Helmholtz equation. We restrict our study here to a Dirichlet (for $g=-\gamma_{0}^{+} u^{\text {inc }}$ ) or a Neumann (for $g=-\gamma_{1}^{+} u^{\text {inc }}$ ) boundary condition, where $\gamma_{j}^{+}$is the exterior trace of order $j$ (see (4)). For acoustic radiation problems, these boundary conditions respectively correspond to the scattering problem by a sound-soft or a sound-hard body. Finally, the well-known Sommerfeld radiation condition at infinity is imposed to ensure the uniqueness of the solution to the boundary value problem (1).

We can associate to (1) the following interior boundary value problem

$$
\left\{\begin{array}{l}
\text { Find } u^{-} \in H^{1}\left(\Omega^{-}\right) \text {such that } \\
\Delta u^{-}+k^{2} u^{-}=0, \text { in } \mathcal{D}^{\prime}\left(\Omega^{-}\right), \\
\gamma_{j}^{-} u^{-}=g, \text { in } H^{1 / 2-j}(\Gamma), \quad \text { for } j=0 \text { or } 1
\end{array}\right.
$$

Let us introduce the Sobolev spaces

$$
\begin{aligned}
& H_{-}^{1}(\Delta):=H^{1}\left(\Delta, \overline{\Omega^{-}}\right):=\left\{u \in H^{1}\left(\overline{\Omega^{-}}\right) ; \Delta u \in L^{2}\left(\overline{\Omega^{-}}\right)\right\}, \\
& H_{+}^{1}(\Delta):=H_{\mathrm{loc}}^{1}\left(\Delta, \overline{\Omega^{+}}\right):=\left\{u \in H_{\mathrm{loc}}^{1}\left(\overline{\Omega^{+}}\right) ; \Delta u \in L_{\mathrm{loc}}^{2}\left(\overline{\Omega^{+}}\right)\right\} .
\end{aligned}
$$

Then, for $u^{ \pm} \in H_{ \pm}^{1}(\Delta)$, the exterior $(+)$ and interior $(-)$ trace operators of order $j(j=0$ or 1$)$ can be both defined by

$$
\begin{aligned}
\gamma_{j}^{ \pm}: H_{ \pm}^{1}(\Delta) & \rightarrow H^{1 / 2-j}(\Gamma) \\
u^{ \pm} & \mapsto \gamma_{j}^{ \pm} u^{ \pm}=\partial_{\mathbf{n}}^{j} u_{\mid \Gamma}^{ \pm} .
\end{aligned}
$$

Vector $\mathbf{n}$ is the outwardly directed unit vector to $\Omega^{-}$.

One of the main difficulties arising in the solution of an exterior boundary value problem is related to the unboundedness of the domain $\Omega^{+}$. To overcome this inconvenient, the integral equation method is used [26,29]. This technique consists in rewriting the initial problem (1) equivalently as an integral equation set on the finite 
surface $\Gamma$. The first step is based on the integral representation of the exterior field $u^{+}$as the superposition of the single- and double-layer potentials $\mathcal{L}$ and $\mathcal{M}$ as follows

$$
u^{+}(x)=-\mathcal{M} \gamma_{0}^{+} u^{+}(x)-\mathcal{L} \gamma_{1}^{+} u^{+}(x), \quad \forall x \in \Omega^{+} .
$$

Therefore, the Cauchy data $\left(\gamma_{0}^{+} u^{+}, \gamma_{1}^{+} u^{+}\right)$become the new unknowns. The interior field is expressed as

$$
u^{-}(x)=\mathcal{M} \gamma_{0}^{-} u^{-}(x)+\mathcal{L} \gamma_{1}^{-} u^{-}(x), \quad \forall x \in \Omega^{-} .
$$

The integral operators $\mathcal{L}$ and $\mathcal{M}$ are respectively given by

$$
\begin{aligned}
& \mathcal{L} p(x)=\int_{\Gamma} G(x, y) p(y) \mathrm{d} \Gamma(y), \quad x \notin \Gamma, \\
& \mathcal{M} \phi(x)=-\int_{\Gamma} \partial_{\mathbf{n}(y)} G(x, y) \phi(y) \mathrm{d} \Gamma(y), \quad x \notin \Gamma
\end{aligned}
$$

for two well-defined densities $p$ and $\phi$. Function $G$ stands for the Helmholtz's kernel

$$
G(x, y)=\frac{1}{4 \pi} \frac{\mathrm{e}^{i k|x-y|}}{|x-y|}, \quad x \neq y .
$$

Since our goal is to write an integral equation on $\Gamma$, we have to express the first two traces of $u^{ \pm}$. To this end, we use the following result [45].

Proposition 2.1. Under the previous notations, the first two traces of $u^{ \pm} \in H_{ \pm}^{1}(\Delta)$ are related by the integral representations

$$
\gamma_{0}^{ \pm} u^{ \pm}=\left( \pm \frac{I}{2}-M\right) \gamma_{0}^{ \pm} u^{ \pm}-L \gamma_{1}^{ \pm} u^{ \pm}, \quad \text { in } H^{1 / 2}(\Gamma)
$$

and

$$
\gamma_{1}^{ \pm} u^{ \pm}=\left( \pm \frac{I}{2}-N\right) \gamma_{1}^{ \pm} u^{ \pm}-D \gamma_{0}^{ \pm} u^{ \pm}, \quad \text { in } H^{-1 / 2}(\Gamma)
$$

where $I$ is the identity operator.

The operators $L$ and $M$ are respectively the trace of the single- and double-layer potentials

$$
\begin{aligned}
& L p(x)=\int_{\Gamma} G(x, y) p(y) \mathrm{d} \Gamma(y), \quad \forall x \in \Gamma, \\
& M \phi(x)=-\int_{\Gamma} \partial_{\mathbf{n}(y)} G(x, y) \phi(y) \mathrm{d} \Gamma(y), \quad \forall x \in \Gamma,
\end{aligned}
$$

and the operators $N$ and $D$ respectively the normal derivative trace of the single- and double-layer potentials

$$
\begin{aligned}
& N p(x)=\partial_{\mathbf{n}(x)} \int_{\Gamma} G(x, y) p(y) \mathrm{d} \Gamma(y)=-M^{t} p, \quad \forall x \in \Gamma, \\
& D \phi(x)=-\partial_{\mathbf{n}(x)} \int_{\Gamma} \partial_{\mathbf{n}(y)} G(x, y) \phi(y) \mathrm{d} \Gamma(y), \quad \forall x \in \Gamma .
\end{aligned}
$$

In the above relations, $A^{t}$ designates the transposed operator of an operator $A$. These boundary integral operators define the following continuous mappings [45]

$$
\begin{array}{lllll}
L & : & H^{-1 / 2}(\Gamma) & \rightarrow & H^{1 / 2}(\Gamma) \\
M & : & H^{1 / 2}(\Gamma) & \rightarrow & H^{1 / 2}(\Gamma) \\
N & : & H^{-1 / 2}(\Gamma) & \rightarrow & H^{-1 / 2}(\Gamma) \\
D & : & H^{1 / 2}(\Gamma) & \rightarrow & H^{-1 / 2}(\Gamma)
\end{array}
$$


For a smooth surface, the double-layer operator $M$ is compact from $H^{1 / 2}(\Gamma)$ into $H^{1 / 2}(\Gamma)$. In the case of a less regular boundary (for instance a Lipschitz boundary), this property is no longer satisfied. A possible approach to rigorously extend the developments of the present paper could be to use some regularizing techniques as in the recent paper of Buffa and Hiptmair [15].

\section{Generalized Brakhage-Werner integral formulations}

Different ways of designing integral equations for solving (1) exist. We propose here to construct some new second-kind Fredholm integral equations well-suited for the iterative solution by a Krylov solver. Among the most widely used formulations are the Brakhage-Werner integral formulations. These equations are based on the representation of the scattered field as a linear combination of the single- and double-layer potentials applied to some non-physical densities. We propose here a general background for generalizing such formulations.

Firstly, let us consider the case of a Dirichlet boundary condition and let us start with the exterior integral representation $(7)$

$$
\left(\frac{I}{2}-M\right) \gamma_{0}^{+} u^{+}-L\left(\gamma_{1}^{+} u^{+}\right)=g, \quad \text { in } H^{1 / 2}(\Gamma)
$$

A well-known result is that the integral equation resulting from replacing $\gamma_{0}^{+} u^{+}$by $g$ and solved according to the normal derivative trace is ill-posed at resonant frequencies. For this reason, formulations based on combined single- and double-layer potentials are rather preferred like for instance the ones proposed by Brakhage-Werner (BW) [29]. More precisely, their approach consists in determining an unknown density $\psi$ solution to the BW equation

$$
\left(\frac{I}{2}-M-\eta L\right) \psi=g, \text { in } H^{1 / 2}(\Gamma) .
$$

These formulations are well-posed for any wavenumber $k$ as long as the coupling parameter $\eta$ has a positive imaginary part [29]. Moreover, a numerical study due to Kress [40] shows that an "almost optimal" coupling parameter $\eta$ yielding a minimal condition number of the operator defining (13) is $\eta=i k$. These formulations can be generalized as follows [6]. Let us assume that the exact Dirichlet-Neumann (DN) map $\boldsymbol{\Lambda}^{\text {ex }}$ given by [7]

$$
\begin{aligned}
& \boldsymbol{\Lambda}^{\mathrm{ex}}: H^{1 / 2}(\Gamma) \rightarrow H^{-1 / 2}(\Gamma) \\
& \gamma_{0}^{+} u^{+} \quad \mapsto \gamma_{1}^{+} u^{+}=\boldsymbol{\Lambda}^{\mathrm{ex}} \gamma_{0}^{+} u^{+} \text {, }
\end{aligned}
$$

is known. Then, the exterior normal derivative trace can be directly computed from $\gamma_{1}^{+} u^{+}=\boldsymbol{\Lambda}^{\mathrm{ex}} g$. Moreover, from (12) and (14) the following identity holds

$$
\frac{I}{2}-M-L \Lambda^{\mathrm{ex}}=I
$$

However, one generally cannot compute the DN operator for a general surface. The idea proposed in [6] consists in considering some suitable analytic approximations of the DN operator. Essentially, we consider an operator $\widetilde{\boldsymbol{\Lambda}}$ approximating the exact operator $\boldsymbol{\Lambda}^{\text {ex }}$ and we solve the integral equation for an unknown density $\psi$

$$
B_{D} \psi=g \quad \text { in } H^{1 / 2}(\Gamma),
$$

setting

$$
B_{D}=\left(\frac{I}{2}-M\right)-L \tilde{\Lambda}
$$

A suitable choice of $\widetilde{\boldsymbol{\Lambda}}$ results in a second-kind Fredholm integral operator well-suited for a Krylov solution. The exterior normal derivative of the field can be reconstructed from $\psi$

$$
\gamma_{1}^{+} u^{+}=\left(\frac{I}{2}-N\right) \widetilde{\boldsymbol{\Lambda}} \psi-D \psi,
$$


using Proposition 2.1. Moreover, choosing $\widetilde{\boldsymbol{\Lambda}}=i k$ (Sommerfeld radiation condition) yields the parameter of Kress [6]. Therefore, this approach can be seen as a generalization of the one proposed by Brakhage and Werner.

For a Neumann boundary condition, the starting point is based on the following integral formulation (see Eq. (8))

$$
\left(\frac{I}{2}-N\right) \gamma_{1}^{+} u^{+}-D \gamma_{0}^{+} u^{+}=g, \quad \text { in } \quad H^{-1 / 2}(\Gamma)
$$

This equation suffers from spurious internal resonances if the normal derivative trace is replaced by $g$ and the equation is solved according to the exterior trace $\gamma_{0}^{+} u^{+}$. To construct a well-posed equation, let us suppose that a suitable approximation $\widetilde{\mathbf{V}}$ of the exact Neumann-Dirichlet (ND) operator $\mathbf{V}^{\text {ex }}$ can be derived. Then, one solves this equation

$$
B_{N} \varphi=g, \text { in } H^{-1 / 2}(\Gamma)
$$

with

$$
B_{N}=\left(\frac{I}{2}-N\right)-D \tilde{\mathbf{V}}
$$

The exterior trace of the solution can be computed from $\varphi$ by the integral representation (7)

$$
\gamma_{0}^{+} u^{+}=\left(\frac{I}{2}-M\right) \tilde{\mathbf{V}} \varphi-L \varphi
$$

Considering the lowest order approximation $\widetilde{\mathbf{V}}=-i / k$ (Sommerfeld radiation condition) gives the usual BW integral equation with the parameter of Kress. In the sequel, equations (15)-(16) and (18)-(19) are referred to as generalized BW integral equations for respectively the Dirichlet and Neumann boundary conditions.

\section{An approximation of the DN AND ND operators By the BPM}

During the construction of the integral operators $B_{D}$ and $B_{N}$, we have seen that an approximation of the DN and ND pseudodifferential operators must be derived. We propose here to follow some ideas inspired from the Beam Propagation Methods (BPM) usually employed in guided optics or underwater acoustic wave propagation.

Since the boundary $\Gamma$ is a compact manifold, we can describe the surface by a collection of coordinates charts. To this end, let us consider a point $x$ of $\Gamma$. Let $\delta$ be a positive real parameter. A neighborhood $\mathcal{U}_{\delta}$ of $\Gamma$ is given by

$$
\mathcal{U}_{\delta}=\left\{x \in \mathbb{R}^{3} ; \operatorname{dist}(x, \Gamma)<\delta\right\},
$$

where $\operatorname{dist}(x, \Gamma)$ designates the distance of a point $x$ to $\Gamma$. Then, for a sufficiently small value of $\delta$, it can be proved that for any $x \in \mathcal{U}_{\delta}$, there exists a unique $p$ in $\Gamma$ such that: $|p-x|=\delta$. The point $p$ is the orthogonal projection of $x$ onto $\Gamma$. The outwardly unitary vector $\mathbf{n}$ to $\Gamma$ can be locally extended to $\mathcal{U}_{\delta}$. Therefore, we get a natural parametrization of $\mathcal{U}_{\delta}$ given by

$$
\mathcal{U}_{\delta}=\{x=p+r \mathbf{n}(p) ;-\delta<r<\delta, p \in \Gamma\}
$$

More precisely, for $\delta$ small enough, the map $(p, r) \mapsto x=p+r \mathbf{n}(p)$ defines a $\mathcal{C}^{\infty}$ diffeomorphism between $\Gamma \times(-\delta, \delta)$ and $\mathcal{U}_{\delta}$. For $r$ in the interval $]-\delta, \delta\left[\right.$, the parallel surface $\Gamma_{r}$ to $\Gamma$ can be described as $\Gamma_{r}=\{x \in$ $\left.\mathbb{R}^{3} ; x=p+r \mathbf{n}(p)\right\}$. The unitary normal vector to $\Gamma_{r}$ at a point $x$ can be defined by the extension of $\mathbf{n}(x)$ and $\Gamma_{0}=\Gamma$. Let us consider a local chart $(\mathcal{V}, \Psi)$ of $\Gamma: \Psi: \mathcal{V} \rightarrow \Gamma$. Each point $p$ of $\Psi(\mathcal{V})$ is described by $p=\Psi(s)$. We can define the normal vector to $\Gamma$ using the following rule. The chart $(\mathcal{V}, \Psi)$ gives rise to a basis $\left(\boldsymbol{\tau}_{1}, \boldsymbol{\tau}_{2}\right)$ of the tangent plane $T_{p}(\Gamma)$ given by: $\boldsymbol{\tau}_{j}=\partial_{s_{j}} \Psi$, for $j=1,2$, and $s_{j}$ being the coordinates of $s$. We assume that the chart is compatible with the orientation of $\mathbf{n}$, i.e.

$$
\mathbf{n}(p)=\frac{\boldsymbol{\tau}_{1} \wedge \boldsymbol{\tau}_{2}}{\left|\boldsymbol{\tau}_{1} \wedge \boldsymbol{\tau}_{2}\right|}
$$


where $\mathbf{a} \wedge \mathbf{b}$ stands for the usual vector product of two vectors $\mathbf{a}$ and $\mathbf{b}$ in $\mathbb{C}^{3}$. From now on, we make the following choice of local chart. We choose $(\mathcal{V}, \Psi)$ such that the coordinates system $(r, s)$ is orthonormal $(i$.e. $\left(\boldsymbol{\tau}_{1}, \boldsymbol{\tau}_{2}\right)$ is an orthonormal basis of the tangent plane) and such that $\boldsymbol{\tau}_{j}$ is an eigenvector of the curvature tensor. Then, it can be shown (see for instance [7]) that in this principal basis, the Helmholtz equation reads

$$
\partial_{r}^{2} u+2 \mathcal{H}_{r} u+\frac{1}{h_{1} h_{2}}\left(\partial_{s_{1}}\left(\frac{h_{2}}{h_{1}} \partial_{s_{1}} u\right)+\partial_{s_{2}}\left(\frac{h_{1}}{h_{2}} \partial_{s_{2}} u\right)\right)+k^{2} u=0, \text { on } \Gamma_{r}
$$

Hereabove, we have denoted by $h_{j}$ the parameter $h_{j}(r, s)=1+\kappa_{j} r$, for $j=1,2$, where the functions $\kappa_{j}$ designate the principal curvatures of the surface. The mean curvature $\mathcal{H}_{r}$ is given by $\mathcal{H}_{r}=h_{1}^{-1} h_{2}^{-1} \partial_{r}\left(h_{1} h_{2}\right) / 2$.

Since we have a time dependence according to $\mathrm{e}^{-i \omega t}$, then the Beam Propagation Method (BPM) gives that the solution to (20) can be approximated by the square-root operator

$$
\varphi_{r}=i \sqrt{\frac{1}{h_{1} h_{2}}\left(\partial_{s_{1}}\left(\frac{h_{2}}{h_{1}} \partial_{s_{1}}\right)+\partial_{s_{2}}\left(\frac{h_{1}}{h_{2}} \partial_{s_{2}}\right)\right)+k^{2}} \psi_{r}, \text { on } \Gamma_{r}
$$

with $\left(\psi_{r}, \varphi_{r}\right)=\left(\left.u^{+}\right|_{\Gamma_{r}},\left.\partial_{r} u^{+}\right|_{\Gamma_{r}}\right)$. Taking the value of this expression for $r=0$ yields the following approximation of the DN map on $\Gamma$ through the square-root surface operator

$$
\varphi=i k \sqrt{1+\frac{\Delta_{\Gamma}}{k^{2}}} \psi, \text { on } \Gamma
$$

denoting by $(\psi, \varphi)$ an approximation of the exact Cauchy data $\left(\gamma_{0}^{+} u, \gamma_{1}^{+} u\right)$. The square-root $\sqrt{z}$ of a complex number $z$ stands for the classical complex square-root with branch-cut along the negative real axis. The operator $\Delta_{\Gamma}$ is the usual Laplace-Beltrami operator over the surface $\Gamma$. Since $\Gamma$ is a compact manifold, there exists an orthonormal basis $\left(\phi_{j}\right)_{j \in \mathbb{N}}$ of eigenvectors of $\Delta_{\Gamma}$ associated to the non-negative real eigenvalues $\left(\mu_{j}\right)_{j \in \mathbb{N}}$ and satisfying the eigenvalue problem

$$
-\Delta_{\Gamma} \phi=\mu \phi
$$

Then the square-root operator $\Lambda: H^{1 / 2}(\Gamma) \rightarrow H^{-1 / 2}(\Gamma)$ given by

$$
\boldsymbol{\Lambda}=i k \sqrt{1+\frac{\Delta_{\Gamma}}{k^{2}}}
$$

is defined by the spectral decomposition

$$
\boldsymbol{\Lambda} \psi=i k \sum_{j=1}^{+\infty} \sqrt{1-\frac{\mu_{j}}{k^{2}}} \psi_{j} \phi_{j}, \quad \text { with } \psi=\sum_{j=1}^{+\infty} \psi_{j} \phi_{j}
$$

The modes $j$ such that $\mu_{j}<k^{2}$ correspond to the propagative modes while the ones given for $\mu_{j}>k^{2}$ are linked to the evanescent modes. For scattering problems, a transition region corresponding to modes such that $\mu_{j} \approx k^{2}$ gives the grazing modes. It can be shown $[9,16]$ that the artificial singularity of the square-root operator does not yield a satisfactory representation of these modes. The transition from the propagative modes to the evanescent ones should be a priori smooth. To approximately model this behaviour, we use [9,31] a regularization of $\boldsymbol{\Lambda}$ introducing a small local damping parameter $\varepsilon>0$ in the transition region. More precisely, we consider the following approximation of the DN operator

$$
\varphi=\Lambda_{\varepsilon} \psi
$$


with

$$
\boldsymbol{\Lambda}_{\varepsilon}=i k \sqrt{1+\frac{\Delta_{\Gamma}}{k_{\varepsilon}^{2}}},
$$

setting $k_{\varepsilon}=k+i \varepsilon$. To get a good accuracy for the condition (21)-(22), an "optimal" damping coefficient is given by $\varepsilon=0.4 k^{1 / 3} \mathcal{H}^{2 / 3}$ in [31], setting $\mathcal{H}$ as the mean curvature of the surface $\Gamma$. For the sake of simplicity, we roughly choose here $\varepsilon=0.4 k^{1 / 3} R^{-2 / 3}$ with $R$ the radius of the smallest sphere containing the scatterer. We will see later ( $c f$. Sect. 9) that this choice is sufficient and leads to an optimal convergence of the resulting iterative algorithm.

Concerning the approximation of the ND operator $\mathbf{V}^{\text {ex }}$, we naturally choose

$$
\begin{aligned}
\mathbf{V}_{\varepsilon}: H^{-1 / 2}(\Gamma) & \rightarrow H^{1 / 2}(\Gamma) \\
\varphi & \mapsto \psi=\mathbf{V}_{\varepsilon} \varphi,
\end{aligned}
$$

setting

$$
\mathbf{V}_{\varepsilon}=\frac{1}{i k}\left(1+\frac{\Delta_{\Gamma}}{k_{\varepsilon}^{2}}\right)^{-1 / 2}
$$

\section{Solvability of the Generalized BW integral EQUATions}

The classical BW integral equations have been constructed to be uniquely solvable [29]. We propose here to prove that this is also the case for their generalizations incorporating the regularized square-root operator.

\subsection{A preliminary result}

Before giving the proof, we state a preliminary result concerning the computation of the principal symbol of the single-layer potential $L$ and the normal derivative of the double-layer potential $D$.

Lemma 5.1. Let $L$ and $D$ be respectively the single-layer and normal derivative trace of the double-layer potentials defined by the expressions (5) and (10). Let $\xi$ be the dual variable of $x$ by Fourier transform for $x$ restricted to $\Gamma$. Then, the principal symbols of $L$ and $D$, denoted by $\sigma_{p}(L)$ and $\sigma_{p}(D)$, are given by

$$
\sigma_{p}(L)=\frac{i}{2 \sqrt{k^{2}-|\xi|^{2}}} \quad \text { and } \quad \sigma_{p}(D)=\frac{\sqrt{k^{2}-|\xi|^{2}}}{2 i} .
$$

Proof. We do not detail the proof. Essentially, the arguments follow the ones given e.g. in [20] (pp. 88-93) for the Laplace operator. The main difference is that the presence of the wavenumber in the definition of the Helmholtz operator leads to incorporate $k$ in the calculation of the principal symbol.

Remark. The coefficient $k$ can be seen as the dual variable of the time variable. Its introduction in the calculus of the principal symbol is a key argument for stating the following existence and uniqueness results.

\subsection{The sound-soft scattering problem}

The generalized BW integral equation for the Dirichlet boundary condition is given by

$$
B_{D, \varepsilon} \psi=g, \text { in } H^{1 / 2}(\Gamma),
$$

where the integral operator $B_{D, \varepsilon}: H^{1 / 2}(\Gamma) \rightarrow H^{1 / 2}(\Gamma)$ is defined by $B_{D, \varepsilon}=(I / 2-M)-L \boldsymbol{\Lambda}_{\varepsilon}$. The operators $M$ and $L$ are given by the representations (5). The square-root operator $\boldsymbol{\Lambda}_{\varepsilon}$ is expressed in (22). Then, the following result holds.

Proposition 5.2. The integral equation (26) is uniquely solvable for any frequency $k>0$ and damping parameter $\varepsilon>0$. 
Proof. Let $\Gamma$ be a smooth surface. The first point consists in stating that the operator $B_{D, \varepsilon}$ can be written as $a I+K$, where $a \neq 0$ is a constant and $K$ a compact operator from $H^{1 / 2}(\Gamma)$. From Lemma 5.1 and the expression (22) of the square-root operator $\boldsymbol{\Lambda}_{\varepsilon}$, we straightforwardly have the respective principal symbol of these operators

$$
\sigma_{p}(L)=\frac{-1}{2|\xi| \sqrt{1-k^{2} /|\xi|^{2}}} \text { and } \sigma_{p}\left(\boldsymbol{\Lambda}_{\varepsilon}\right)=\frac{k}{k_{\varepsilon}}|\xi| \sqrt{1-\frac{k_{\varepsilon}^{2}}{|\xi|^{2}}} .
$$

Writing the series expansion of these two symbols using $(1-z)^{-1 / 2}$ for $z=k^{2} /|\xi|^{2}$ and $(1-z)^{1 / 2}$ for $z=k_{\varepsilon}{ }^{2} /|\xi|^{2}$ in the elliptic part of the operators (for $|\xi| \rightarrow+\infty$ ), we obtain

$$
\sigma_{p}(L)=-\frac{1}{2|\xi|}\left(1+\sum_{\ell=1}^{+\infty} \frac{L_{\ell}}{|\xi|^{2 \ell}}\right) \text { and } \sigma_{p}\left(\boldsymbol{\Lambda}_{\varepsilon}\right)=\frac{k}{k_{\varepsilon}}|\xi|\left(1+\sum_{\ell=1}^{+\infty} \frac{\lambda_{\ell}}{|\xi|^{2 \ell}}\right)
$$

for some complex numbers $L_{\ell}$ and $\lambda_{\ell}$ depending on $\ell \in \mathbb{N}^{*}, k$ and $\varepsilon$. This implies that the principal symbol of the composition of these two operators can be written as

$$
\sigma_{p}\left(-L \boldsymbol{\Lambda}_{\varepsilon}\right)=\frac{k}{2 k_{\varepsilon}}+\sum_{\ell=1}^{+\infty} \frac{d_{\ell}}{|\xi|^{2 \ell}}
$$

for some complex coefficients $d_{\ell}$, with $\ell \in \mathbb{N}^{*}$. Finally, the operator $-L \boldsymbol{\Lambda}_{\varepsilon}$ takes the form

$$
-L \boldsymbol{\Lambda}_{\varepsilon}=\frac{k}{2 k_{\varepsilon}} I+\widetilde{K}
$$

with $\widetilde{K}$ a compact operator of negative order. (This relation can be compared in a certain sense to the Calderón's relation $-2 L D=I / 2-2 M^{2}$ used by Christiansen and Nédélec $\left.[27,28]\right)$. Returning to the operator $B_{D, \varepsilon}$, we deduce from the above result that

$$
B_{D, \varepsilon}=a I+K
$$

with the complex number $a=1 / 2+k /\left(2 k_{\varepsilon}\right) \neq 0$ for $k>0$ and $K=-M+\widetilde{K}$ a compact perturbation since it is the sum of two compact operators from $H^{1 / 2}(\Gamma)$.

Since the operator $B_{D, \varepsilon}$ arising in (26) is a second-kind Fredholm operator, the Riesz-Fredholm theory classically yields its inversibility if the operator is injective. Therefore, it is sufficient to prove that the homogeneous equation associated to (26) admits $\psi=0$ as unique solution to get the existence and uniqueness of the solution to the generalized BW integral equation (26). Let $\psi \in H^{1 / 2}(\Gamma)$ solution to $B_{D, \varepsilon} \psi=0$. Then, the function $u^{+}$ defined by

$$
u^{+}(x)=-\mathcal{M} \psi(x)-\mathcal{L} \boldsymbol{\Lambda}_{\varepsilon} \psi(x), \quad \forall x \in \Omega^{+},
$$

is solution to the homogeneous exterior Dirichlet problem and $u^{+}=0$ in $\Omega^{+}$. Using Proposition 2.1, one gets

$$
-\gamma_{0}^{-} u^{-}=\psi \text {, in } H^{1 / 2}(\Gamma) \text { and } \quad-\gamma_{1}^{-} u^{-}=\boldsymbol{\Lambda}_{\varepsilon} \psi \text {, in } H^{-1 / 2}(\Gamma) \text {. }
$$

The first Green formula in the interior domain $\Omega^{-}$gives

$$
\left\langle\gamma_{0}^{-} u^{-}, \gamma_{1}^{-} u^{-}\right\rangle_{\Gamma}=\int_{\Omega^{-}}\left|\nabla u^{-}\right|^{2}-k^{2}\left|u^{-}\right|^{2} \mathrm{~d} \Omega^{-}
$$

where $\langle f, g\rangle_{\Gamma}$ is the duality product of two functions $f \in H^{1 / 2}(\Gamma)$ and $g \in H^{-1 / 2}(\Gamma)$. If we take the imaginary part of this equation, we obtain the relation $\Im\left(\left\langle\psi, \Lambda_{\varepsilon} \psi>_{\Gamma}\right)=0\right.$. From the definition of the square-root 
operator $\boldsymbol{\Lambda}_{\varepsilon}$, we have the spectral decomposition

$$
\boldsymbol{\Lambda}_{\varepsilon} \psi=i k \sum_{j=1}^{+\infty} \sqrt{1-\frac{\mu_{j}}{k_{\varepsilon}^{2}}} \psi_{j} \phi_{j}, \quad \text { with } \psi=\sum_{j=1}^{+\infty} \psi_{j} \phi_{j} .
$$

Since $\left\{\phi_{j}\right\}_{j \in \mathbb{N}^{*}}$ is an orthonormal basis, we obtain that the imaginary part is given by

$$
\Im\left(<\psi, \boldsymbol{\Lambda}_{\varepsilon} \psi>_{\Gamma}\right)=k \sum_{j=1}^{+\infty} \Re\left(\sqrt{1-\frac{\mu_{j}}{k_{\varepsilon}^{2}}}\right)\left|\psi_{j}\right|^{2}=0 .
$$

Using that $\mu_{j}>0$ for $j \in \mathbb{N}^{*}$, we can easily prove that $\Re\left(\sqrt{1-\mu_{j} / k_{\varepsilon}^{2}}\right)>0$ for $k>0$ and $\varepsilon>0$. Finally, relation (28) gives that $\psi_{j}=0$ for all $j \in \mathbb{N}^{*}$ and hence $\psi=0$. This ends the proof of Proposition 5.2.

\subsection{The sound-hard scattering problem}

The generalized BW integral equation to solve for the Neumann problem is written as

$$
B_{N, \varepsilon} \varphi=g, \text { in } H^{-1 / 2}(\Gamma)
$$

with $B_{N, \varepsilon}=I / 2-N-D \mathbf{V}_{\varepsilon}$ acting from $H^{-1 / 2}(\Gamma)$ to $H^{-1 / 2}(\Gamma)$. Then, we have the existence and uniqueness of the solution to this equation.

Proposition 5.3. The generalized BW integral equation (29) admits one and only one solution for any wavenumber $k>0$ and damping parameter $\varepsilon>0$.

Proof. We do not detail the proof which follows similar arguments as for the Dirichlet problem.

One of the most important properties of the integral equation (29) is its second-kind nature. Indeed, since the operator $D$ is a first-order pseudodifferential operator and $\mathbf{V}_{\varepsilon}$ is a pseudodifferential operator of order -1 whose principal symbol is almost the inverse of $\sigma_{p}(D), D \mathbf{V}_{\varepsilon}$ is a second-kind integral operator. As seen below, this property is essential from a numerical point of view since second-kind integral equations of the form $a I+K$ with $a \neq 0$ and $K$ compact are characterized by a cluster of eigenvalues around $a$ for $|\xi| \rightarrow+\infty$. This configuration is essential for a good behaviour of a Krylov iterative solver as e.g. the GMRES [17,39,47]. This is not the case of the usual BW integral equation because the approximation $-i D / k$ remains a first-order pseudodifferential operator. Let us notice that the situation is different for a Dirichlet boundary condition. The usual BW operator $B_{D}$ is already a compact perturbation of the identity operator. A better eigenvalue clustering is expected for $B_{D, \varepsilon}$ by choosing the operator $\boldsymbol{\Lambda}_{\varepsilon}$. We will see during the numerical experiments that the most visible improvements are given for the Neumann problem.

\section{THE EXAMPLE OF THE SPHERE}

Let $\Gamma=S_{1}$ be the sphere of radius 1 centered at the origin. A classical result [29] shows that $\mu_{\ell}=-\ell(\ell+1)$ are the eigenvalues of $\Delta_{S_{1}}$. The subspace spanned by the eigenfunctions of $\Delta_{S_{1}}$ has a dimension equal to $2 \ell+1$. Let us introduce the spherical harmonics $Y_{\ell m}$ as the functions defined by

$$
Y_{\ell m}(\hat{\theta})=\left[\frac{2 \ell+1}{4 \pi} \frac{(\ell-|m|) !}{(\ell+|m|) !}\right]^{1 / 2} P_{\ell}^{m}(\cos \theta) \mathrm{e}^{i m \phi}
$$

for $\ell=0,1,2, \ldots,-\ell \leq m \leq \ell$, and $P_{\ell}^{m}$ the associated Legendre polynomials. Here, we have defined $(r, \hat{\theta})$ as the spherical polar coordinate system such that $\hat{\theta}=(\theta, \phi) \in S_{1}$. The functions $Y_{\ell m}$, for $\ell=0,1,2, \ldots,-\ell \leq m \leq \ell$, constitute an orthonormal basis for $L^{2}\left(S_{1}\right)$. In addition, they also form a basis of eigenvectors for the four 

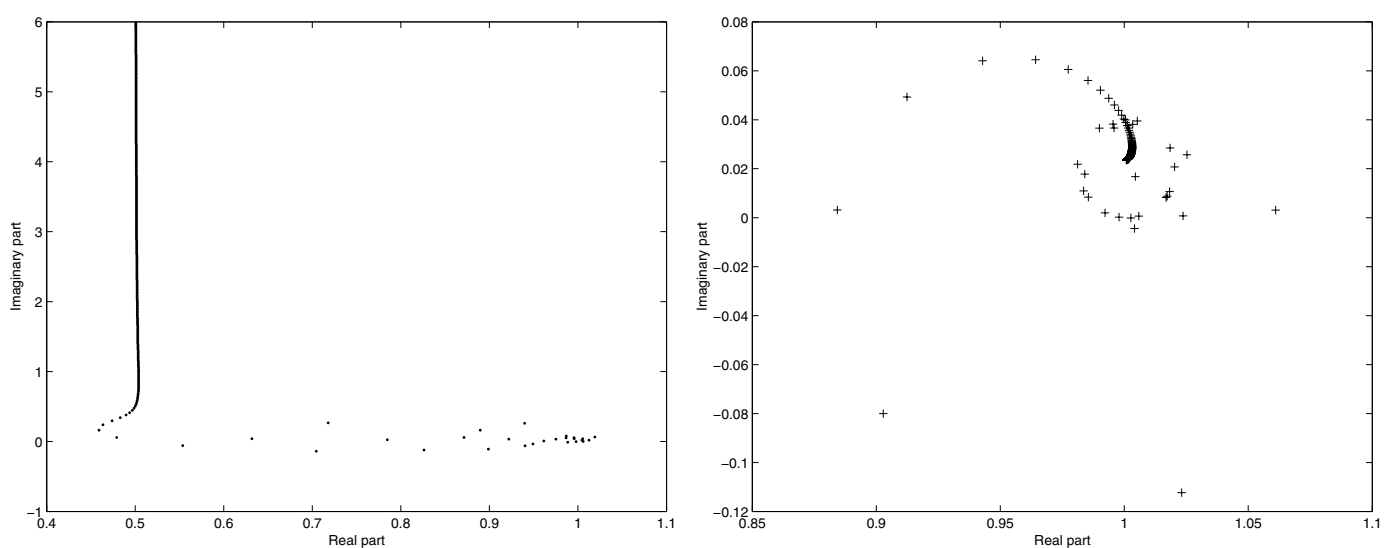

FigurE 1. Sound-hard sphere: eigenvalues of the usual (left) and generalized (right) BrakhageWerner integral operators for a frequency $k=25$ and $n_{\lambda}=24$.

integral operators $L, M, N$ and $D$. If $j_{\ell}$ and $h_{\ell}^{(1)}$ are respectively the spherical Bessel and first-kind Hankel functions of order $\ell$, we have the following proposition (see $[2,40]$ ).

Proposition 6.1. The eigenvalues $\mathcal{L}_{\ell}, \mathcal{M}_{\ell}, \mathcal{N}_{\ell}$ and $\mathcal{D}_{\ell}$ of multiplicity $(2 \ell+1)$ respectively associated with the elementary integral operators $L, M, N$ and $D$ are given by

$$
\begin{aligned}
& L Y_{\ell m}=\mathcal{L}_{\ell} Y_{\ell m}=\left(i k j_{\ell}(k) h_{\ell}^{(1)}(k)\right) Y_{\ell m}, \\
& M Y_{\ell m}=\mathcal{M}_{\ell} Y_{\ell m}=\left(\frac{1}{2}-i k^{2} j_{\ell}^{\prime}(k) h_{\ell}^{(1)}(k)\right) Y_{\ell m}, \quad \mathcal{N}_{\ell} Y_{\ell m}=-\mathcal{M}_{\ell} Y_{\ell m}, \\
& D Y_{\ell m}=\mathcal{D}_{\ell} Y_{\ell m}=\left(-i k^{3} j_{\ell}^{\prime}(k)\left(h_{\ell}^{(1)}\right)^{\prime}(k)\right) Y_{\ell m},
\end{aligned}
$$

for $\ell=0,1,2, \ldots,-\ell \leq m \leq \ell$.

A direct computation gives the eigenvalues $\mathcal{B}_{D, \varepsilon}^{\ell}$ and $\mathcal{B}_{N, \varepsilon}^{\ell}$ of respectively the operators $B_{D, \varepsilon}$ and $B_{N, \varepsilon}$

$$
B_{D, \varepsilon} Y_{\ell m}=\mathcal{B}_{D, \varepsilon}^{\ell} Y_{\ell m}=\left(\left(\frac{1}{2}-\mathcal{M}_{\ell}\right)-\mathcal{L}_{\ell} \boldsymbol{\Lambda}_{\varepsilon \ell}\right) Y_{\ell m}
$$

and

$$
B_{N, \varepsilon} Y_{\ell m}=\mathcal{B}_{N, \varepsilon}^{\ell} Y_{\ell m}=\left(\left(\frac{1}{2}-\mathcal{N}_{\ell}\right)-\mathcal{D}_{\ell} \mathbf{V}_{\varepsilon \ell}\right) Y_{\ell m},
$$

setting $\boldsymbol{\Lambda}_{\varepsilon \ell}=i k \sqrt{1-\mu_{\ell} / k_{\varepsilon}^{2}}$ and $\mathbf{V}_{\varepsilon \ell}=\boldsymbol{\Lambda}_{\varepsilon \ell}^{-1}$, for $\ell=0,1,2, \ldots,-\ell \leq m \leq \ell$.

To illustrate the eigenvalue distribution of the integral operators, we draw in Figure 1 the spectrum of both the usual and generalized BW operators for the unit sphere. For the usual BW integral operator (left figure), we observe a small cluster of eigenvalues linked to the low-order modes (hyperbolic part of the operator [6,31]). A large number of eigenvalues corresponding to the evanescent modes (high-order spatial modes) are on the line $x=1 / 2$. This repartition of eigenvalues penalizes the convergence rate of the GMRES. This problem is avoided with the new operator. Eigenvalue clustering occurs for both the low and high-order harmonics. Just a few eigenvalues corresponding to the coupling between low and high-order modes is observable as a loop around 1. This clustering is characteristic of second-kind Fredholm operators. 


\section{Generalized Combined field integral EQUations}

This section is devoted to a generalization of the Combined Field Integral Equation introduced by Harrington and Mautz in electromagnetism [26,34]. This generalization is particularized to the case of the scattering problem of an electromagnetic wave by a perfectly conducting body for a TM or a TE polarized wave. Therefore, we are led to solve the two-dimensional Helmholtz equation with respectively a Dirichlet or a Neumann boundary condition. The extension to the three-dimensional acoustic problem is simply a transcription of the twodimensional equations. The case of the full Maxwell equations will be studied somewhere else and is beyond the scope of the present paper.

Let us firstly recall some basic results (see e.g. [26]). Let $\mathbf{j}$ be the electric surfacic current representing the jump of the tangential component of the magnetic field through the interface $\Gamma$ of $\Omega^{-}$. By determinating $\mathbf{j}$, we can compute both the electric and magnetic scattered fields from the integral representation theorems for the Maxwell equations. The current $\mathbf{j}$ is solution to the Electric and Magnetic Field Integral Equations (EFIE and MFIE). However, for a closed surface, these integral equations are ill-posed for some resonance frequencies. To overcome this problem, Harrington and Mautz propose to consider a Combined Field Integral Equation (CFIE) resulting from a convex combination between the EFIE and MFIE. More precisely, the usual CFIE is given by

$$
\mathrm{CFIE}=\alpha \mathrm{EFIE}+(1-\alpha) \frac{i}{k} \mathrm{MFIE} .
$$

The coupling parameter $\alpha$ is numerically estimated for minimizing the condition number of the CFIE. We precise different values during the numerical experiments. In the case of a TM polarized wave (Dirichlet), the EFIE and the MFIE are respectively given by

$$
L p=-\gamma_{0}^{+} u^{\text {inc }} \quad \text { and } \quad\left(\frac{I}{2}+N\right) p=-\gamma_{1}^{+} u^{\text {inc }} .
$$

For a TE polarized wave (Neumann), we have the two integral equations

$$
D \phi=-\gamma_{1}^{+} u^{\text {inc }} \quad \text { and } \quad\left(\frac{I}{2}+M\right) \phi=-\gamma_{0}^{+} u^{\text {inc }},
$$

(cf. Sect. 2 for the definitions of the fields $u^{\text {inc }}$ and $u^{ \pm}$.) In the above equations, we have set $p=\gamma_{1}^{+} u^{+}-\gamma_{1}^{-} u^{-}$ and $\phi=\gamma_{0}^{+} u^{+}-\gamma_{0}^{-} u^{-}$. For acoustic field radiation problems, $\phi$ and $p$ respectively represent the jump of the pressure and the normal component of the velocity through the boundary $\Gamma$. Unlike the BW integral formulations, the CFIE hence involves some physical unknowns.

We propose here to generalize the usual CFIE by firstly regularizing the EFIE by the pseudodifferential operator $\boldsymbol{\Lambda}_{\varepsilon}$ or $\mathbf{V}_{\varepsilon}$ according to the polarization and next adding the MFIE. As a by-product, the composition operator resulting from the first step yields a compact perturbation of the identity operator. More precisely, let us consider the Dirichlet boundary value problem. Then, the generalized CFIE is stated as

$$
C_{D, \varepsilon} p=h_{D, \varepsilon}, \quad \text { in } H^{-1 / 2}(\Gamma),
$$

where $C_{D, \varepsilon}$ is the integrodifferential operator defined by

$$
C_{D, \varepsilon}=\left(\frac{I}{2}+N\right)-\Lambda_{\varepsilon} L
$$

and where the right-hand side is given by: $h_{D, \varepsilon}=-\left(\gamma_{1}^{+}-\boldsymbol{\Lambda}_{\varepsilon} \gamma_{0}^{+}\right) u^{\text {inc }}$. We straightforwardly prove that the operator $C_{D, \varepsilon}$ is the Hermitian adjoint of $B_{D, \varepsilon}$ given by (26). Hence, the equation (34) is uniquely solvable at any positive frequency under the same condition as Proposition 5.2. In the usual CFIE (31), a coupling 
parameter $\alpha$ is incorporated in the convex combination. Here, a numerical study shows that $\alpha=0.5$ is the best (natural) choice for the new formulations. For this reason, we do not take this parameter into account.

Concerning the Neumann problem, we consider the following generalization of the CFIE

$$
C_{N, \varepsilon} \phi=h_{N, \varepsilon}, \quad \text { in } H^{1 / 2}(\Gamma),
$$

defining $C_{D, \varepsilon}$ as the operator

$$
C_{N, \varepsilon}=\left(\frac{I}{2}+M\right)-\mathbf{V}_{\varepsilon} D,
$$

and setting: $h_{N, \varepsilon}=-\left(\gamma_{0}^{+}-\mathbf{V}_{\varepsilon} \gamma_{1}^{+}\right) u^{\text {inc }}$. The well-posedness of the equation (35) at any frequency $k>0$ is a consequence of Proposition 5.3 by still noticing that the operator $C_{N, \varepsilon}$ given by (36) is the Hermitian adjoint operator of the integral operator $B_{N, \varepsilon}(29)$. As the usual CFIE, the unknown field $p$ or $\phi$ is a physical quantity unlike the case of the generalized BW integral equations.

\section{Approximation and nUmerical implementation in a KRYlov iterative SOlver}

We only detail here the adopted strategy for the solution of the generalized BW integral equation for the Dirichlet problem which is given by

where the integral operator $B_{D, \varepsilon}$ is

$$
B_{D, \varepsilon} \psi=g, \text { in } H^{1 / 2}(\Gamma),
$$

$$
B_{D, \varepsilon}=\left(\frac{I}{2}-M\right)-L \boldsymbol{\Lambda}_{\varepsilon}
$$

and where $g=-u^{\text {inc }}$ on $\Gamma$. The other kinds of generalized equations can be treated using a similar adapted approach.

Let us introduce a triangulation $\mathcal{T}_{h}=\cup_{j=1}^{N_{T}} T$ of $\Gamma$. The polyedric interpolated surface is denoted by $\Gamma_{h}$. We define the total number of triangles by $N_{T}$ and the total number of vertices by $N_{V}$. Our approximation is based on a linear Galerkin boundary element method even if others approaches can be a priori developed. To this end, let us introduce the following approximation space

$$
V_{h}=\left\{\phi_{h} \in \mathcal{C}^{0}\left(\Gamma_{h}\right) ; \phi_{T}=\phi_{h \mid T} \in \mathbb{P}_{1}, \quad \forall T \in \mathcal{T}_{h}\right\} .
$$

As a consequence, we have $\operatorname{dim} V_{h}=N_{V}$. If $h_{\max }$ is the maximal length of the edges of the triangles involved in $\mathcal{T}_{h}$, then $n_{\lambda}=\lambda / h_{\max }$ designates the density of discretization points per wavelength. In the sequel, if $Z$ is an integral or a differential operator, we denote by $[Z]$ the discretized matrix associated to the linear discretization of $Z$. Following this approximation, the discretization of (37)-(38) leads to the solution of the dense complex non-hermitian linear system of equations

$$
\left[B_{D, \varepsilon}\right] \boldsymbol{\psi}_{h}=[I] \mathbf{g}_{h}, \text { with }\left[B_{D, \varepsilon}\right]=\left(\frac{[I]}{2}-[M]\right)-[L][I]^{-1}\left[\boldsymbol{\Lambda}_{\varepsilon}\right] .
$$

The complex valued vectors $\boldsymbol{\psi}_{h}$ and $\mathbf{g}_{h}$ are elements of $\mathbb{C}^{N_{V}}$. They are respectively the $\mathbb{P}_{1}$-interpolation of the unknown density $\psi$ and of the right hand side $g$. Concerning the evaluation of the integral operators $M$ and $L$, we have used their weak variational formulations while the approximation $[I]$ of the identity operator $I$ is just the mass matrix. A suitable approximation and fast evaluation of the pseudodifferential operator $\boldsymbol{\Lambda}_{\varepsilon}$ is detailled below.

Since we are dealing with the solution of a second-kind Fredholm integral equation [17,39,47], we consider the GMRES procedure without restart $[49,50]$ as Krylov iterative solver for the solution of (39). The GMRES requires one matrix-vector product at each iteration. It is now well-known that a direct evaluation of a matrixvector product needs $\mathcal{O}\left(N_{V}^{2}\right)$ operations (option taken in the paper) and that the Fast Multipole Method (FMM) leads to an efficient evaluation in $\mathcal{O}\left(N_{V} \log N_{V}\right)$ operations. However, we are rather interested here 
in the convergence rate of the GMRES for the different kinds of integral equations. This implies that a good qualitative parameter to measure it is given by the total number of matrix-vector products to get an a priori fixed tolerance $t o l$ in the GMRES. However, this remark is valid if the application of the pseudodifferential operator $\boldsymbol{\Lambda}_{\varepsilon}$ is efficiently realized. Let us now detail both its approximation and implementation.

The discretization of the pseudodifferential operator $\boldsymbol{\Lambda}_{\varepsilon}$ is not straightforward. Moreover, the matrix which corresponds to the numerical discretization of the non-local operator results in a dense complex matrix. Hence, the application of this matrix to a vector $\mathbf{x} \in \mathbb{C}^{N_{V}}$ needs a $\mathcal{O}\left(N_{V}^{2}\right)$ computational cost. To realize a suitable and efficient implementation, we proceed as in the BPM methods using a paraxial approximation of the square-root operator. To this end, we must be able to simulate the pseudodifferential operator $\boldsymbol{\Lambda}_{\varepsilon}=i k \sqrt{1+X}$ for the partial differential operator $X=\Delta_{\Gamma} / k_{\varepsilon}^{2}$ with a small value of $\varepsilon$ and any spatial frequency. We propose to follow the rotating branch-cut technique introduced by Milinazzo et al. [46] for the modeling of underwater acoustic wave propagation and later used in the OSRC context for acoustic scattering [9]. More precisely, this approximation is defined by

$$
\sqrt{1+X} \approx \mathrm{e}^{i \theta / 2} R_{p}\left((1+X) \mathrm{e}^{-i \theta}-1\right)=A_{0}+\sum_{j=1}^{p} \frac{A_{j} X}{1+B_{j} X},
$$

where the complex valued constants $A_{0}, A_{j}$ and $B_{j}$ are given by

$$
A_{0}=\mathrm{e}^{i \theta / 2} R_{p}\left(\mathrm{e}^{-i \theta}-1\right), \quad A_{j}=\frac{\mathrm{e}^{-i \theta / 2} a_{j}}{\left(1+b_{j}\left(\mathrm{e}^{-i \theta}-1\right)\right)^{2}} \quad \text { and } \quad B_{j}=\frac{\mathrm{e}^{-i \theta} b_{j}}{\left(1+b_{j}\left(\mathrm{e}^{-i \theta}-1\right)\right)} .
$$

Hereabove, $R_{p}$ denotes the usual Padé approximant of order $p$ with branch-cut along $\{z \in \mathbb{C} ; z<-1\}$

$$
R_{p}(z)=1+\sum_{j=1}^{p} \frac{a_{j} z}{1+b_{j} z}
$$

setting

$$
a_{j}=\frac{2}{2 p+1} \sin ^{2}\left(\frac{j \pi}{2 p+1}\right) \text { and } b_{j}=\cos ^{2}\left(\frac{j \pi}{2 p+1}\right) .
$$

The angle $\theta$ allows to rotate the branch-cut $\{z \in \mathbb{C} ; z<-1\}$ and to position it along the ray $z=-1+r \mathrm{e}^{i(\theta+\pi)}$. In [31], it is shown that the parameters $p=8$ and $\theta=\pi / 3$ yield a satisfactory approximation of the exact squareroot operator. We are led to solve $p$ Helmholtz-type partial differential equations on $\Gamma$ which are approximated by linear surface finite elements in a variational way [4]. Therefore, at each step of the GMRES, we compute in an approximate way the image $\mathbf{y}$ of a vector $\mathbf{x} \in \mathbb{C}^{N_{V}}$ by $\left[\boldsymbol{\Lambda}_{\varepsilon}\right]$ firstly solving $p$ highly sparse decoupled linear systems (eventually parallelized on different processors)

$$
\left(-\frac{B_{j}}{k_{\varepsilon}^{2}}\left[\Delta_{\Gamma}\right]+[I]\right) \mathbf{x}_{j}=[I] \mathbf{x}, \quad j=1, \ldots, p,
$$

and next computing

$$
\mathbf{y}=i k\left(A_{0} \mathbf{x}-\sum_{j=1}^{p} A_{j}[I]^{-1}\left[\Delta_{\Gamma}\right] \mathbf{x}_{j}\right) .
$$

The matrix $\left[\Delta_{\Gamma}\right]$ represents the surface stiffness matrix. The solution of the $p$ sparse linear systems given by (40) is efficiently obtained by an ILUT preconditioned GMRES with a threshold parameter equal to $10^{-2}$ for a tolerance $10^{-8}$. The convergence for the resolution of each system requires 2 or 3 iterations. Finally, the resulting cost of this procedure is linear and asymptotically equivalent to $\mathcal{O}\left(p N_{V}\right)$. This cost is negligible compared to the usual $\operatorname{cost} \mathcal{O}\left(N_{V} \log N_{V}\right)$ of a matrix-vector product in the FMM since $p$ is relatively small. 


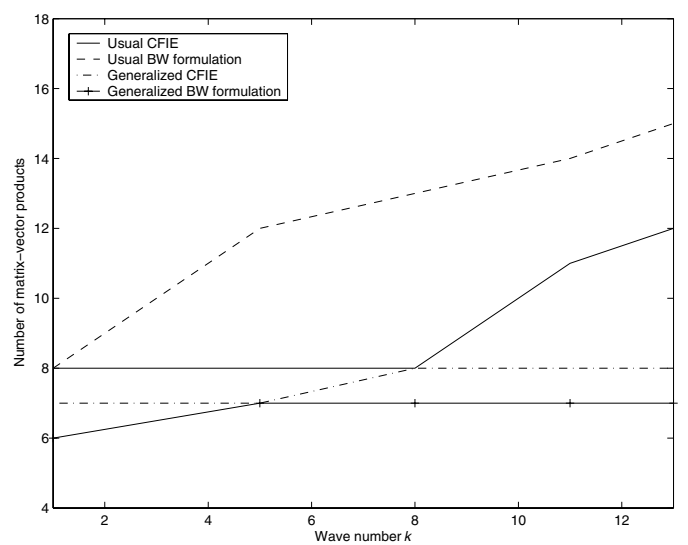

FiguRE 2. Sound-soft unit sphere: number of matrix-vector products required to reach a residual equal to $10^{-6}$ for the four integral formulations with respect to the wavenumber $k$.

\section{Numerical RESUlts}

We present some numerical experiments to compare the different kinds of integral formulations. We consider an incident plane wave

$$
u^{\text {inc }}(x):=\exp (i k \nu \cdot x)
$$

where $\boldsymbol{\nu}=\left(\cos \phi^{\text {inc }} \cos \theta^{\text {inc }}, \cos \phi^{\text {inc }} \sin \theta^{\text {inc }}, \sin \phi^{\text {inc }}\right)$ denotes the vector of incidence in circular or spherical coordinates.

\subsection{The sound-soft scattering problem}

In the case of a Dirichlet datum, we compare the four integral representations: the usual and generalized BW formulations (respectively given by Eq. (13) with $\eta=i k$ and Eq. (26)), the usual and generalized CFIE given by formulations (31)-(32) and (34). The parameter $\alpha$ defining the usual CFIE is taken as $\alpha=0.3$. For a Dirichlet boundary condition, all the integral equations are some second-kind Fredholm equations. Consequently, the condition number does not depend on the discretization step (through for instance the density of discretization points per wavelength) and an eigenvalue clustering occurs for the higher order spatial modes. However, a slight frequency dependence of the condition number should be observable [6,26]. As previously noticed, we consider that the number of matrix-vector products is a good measure to estimate the convergence rate of the GMRES. The GMRES Krylov iterative solver without restart and with a tolerance tol $=10^{-6}$ is used during the numerical simulations. We consider an incident plane wave of incidence zero degree illuminating the soundsoft unit sphere centered at the origin. We report in Figure 2 the evolution of the number of matrix-vector products versus $k$ for a density of discretization points per wavelength $n_{\lambda}=10$. We observe that the generalized formulations exhibit a better behaviour than the usual ones.

\subsection{The sound-hard scattering problem}

Let us now compare the generalized formulations versus the usual integral equations for the sound-hard scattering problem. We take $\alpha=0.2$ in the classical CFIE. In Figures 3 and 4 , we report the resuts corresponding to the scattering of a plane wave of zero degree incidence by both the unit sphere and the ellipsoidal scatterer of semi-axis $a=1$ and $b=c=0.5$ respectively along the $x_{1^{-}}, x_{2^{-}}$and $x_{3^{-}}$-directions and centered at the origin. Once again, we see that the new formulations lead to a better convergence rate of the iterative solver. Unlike the generalized formulations, the number of iterations of the usual formulations depends on the density $n_{\lambda}$. Further, we observe a better behaviour of the generalized formulations than the classical ones according to the wavenumber. 

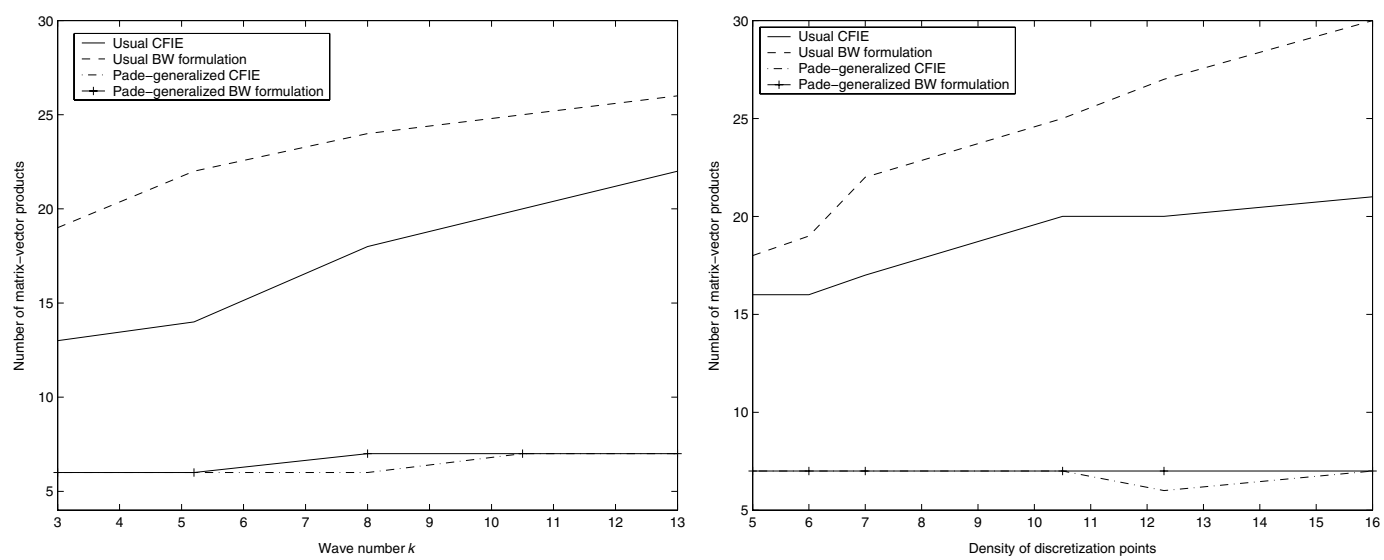

FIGURE 3. Sound-hard unit sphere: number of matrix-vector products required to reach a residual equal to $10^{-6}$ for the four integral formulations with respect to the wavenumber $k$ (left: $n_{\lambda}=10$ and zero degree incidence) and the density of discretization points per wavelength (right: $k=10$ and zero degree incidence).
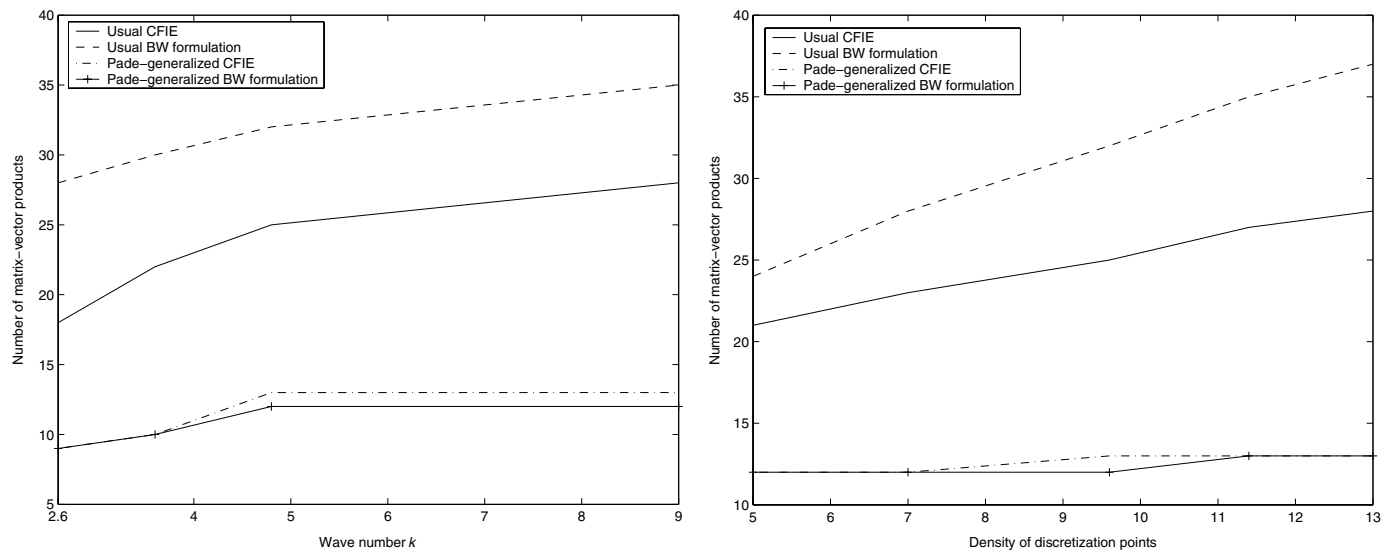

Figure 4. Sound-hard ellipsoidal scatterer: number of matrix-vector products required to reach a residual equal to $10^{-6}$ for the four integral formulations with respect to the wavenumber $k$ (left: $n_{\lambda}=10$ and zero degree incidence) and the density of discretization points per wavelength (right: $k=5$ and zero degree incidence).

We consider scattering of a plane wave by the cube of sidelength 2 centered at the origin. The angle of incidence is equal to 30 degrees. All the remarks of the two previous cases extend to the cube. Here, 8 or 9 iterations are necessary to obtain the solution independently of both the wavenumber and the mesh refinement. We report in Figure 6 the history of the residual norm according to the number of iterations which still shows the fast convergence rate of the generalized formulations.

Let us give two numerical illustrations of the accuracy of the proposed method. The first test consists in considering the scattering of a plane wave of zero degree incidence by the unit sphere for $k=13$ and $n_{\lambda}=10$. We compare the Radar Cross Section (RCS) in decibels (Db) obtained by the usual and generalized integral equations with the exact analytical solution [10]. The RCS is expressed by

$$
\operatorname{RCS}(\mathcal{V})=10 \log _{10}\left(4 \pi\left|a_{0}(\mathcal{V})\right|^{2}\right),
$$



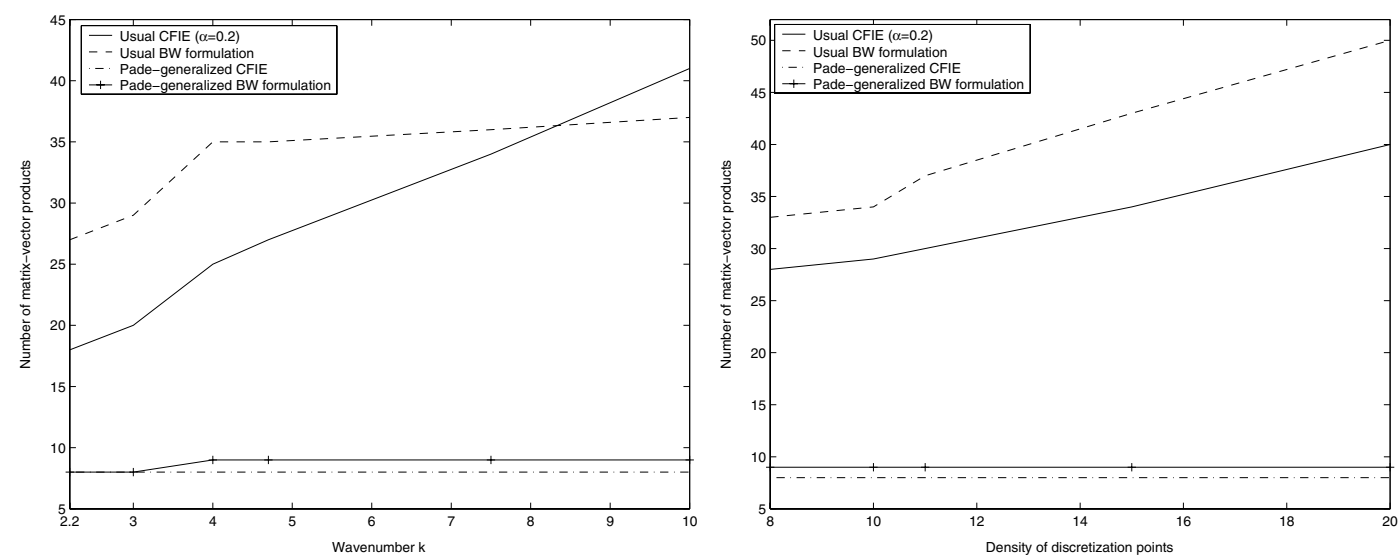

FIGURE 5. Sound-hard unit cube: number of matrix-vector products required to reach a residual equal to $10^{-6}$ for the four integral formulations with respect to the wavenumber $k$ (left: $n_{\lambda}=10$ and incidence $\left.(0,30)\right)$ and the density of discretization points per wavelength (right: $k=5$ and incidence $\left.\left(\phi^{\text {inc }}, \theta^{\text {inc }}\right)=(0,30)\right)$.

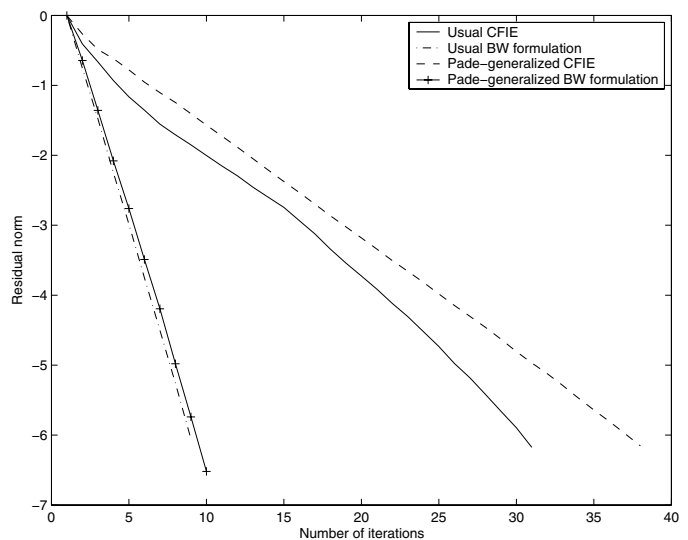

FIgURE 6. Sound-hard unit cube: history of the residual norm according to the number of iterations $\left(k=5, n_{\lambda}=11\right.$ and $\left(\phi^{\text {inc }}, \theta^{\text {inc }}\right)=(0,30)$ degrees incidence $)$.

where $\mathcal{V}$ is the angle of diffusion in the $\left(x_{1}, x_{2}\right)$-plane and $a_{0}(\mathcal{V})$ the scattering amplitude. The second corresponds to the scattering of a plane wave by the unit cube for $k=10$ and $n_{\lambda}=10$. The angle of incidence is $\left(\phi^{\text {inc }}, \theta^{\text {inc }}\right)=(0,30)$. Here, the reference solution is the EFIE which is well-known for its accuracy when the wavenumber $k$ is not a resonant frequency. We obtain the same precision of the numerical solutions.

Finally, we report in Tables 1 and 2 the CPU time and computational cost resulting from the resolution of the auxiliary linear systems compared to the computation of a dense Matrix-Vector Product. We can see clearly that the main cost is related to the MVP calculations and that the solution to the sparse linear system is negligeable. This is more obvious if higher frequencies and refined meshes are used.

\subsection{The case of a non-convex two-dimensional scattering problem}

Let us complete our numerical study with the solution of a two-dimensional scattering problem by a nonconvex object. The obstacle is defined as the square cylinder of sidelength equal to 2 without the square with vertices: $(-1,0.4),(0,0.4),(0,-0.4)$ and $(-1,-0.4)$. The incidence angle being equal to 30 degrees, the 

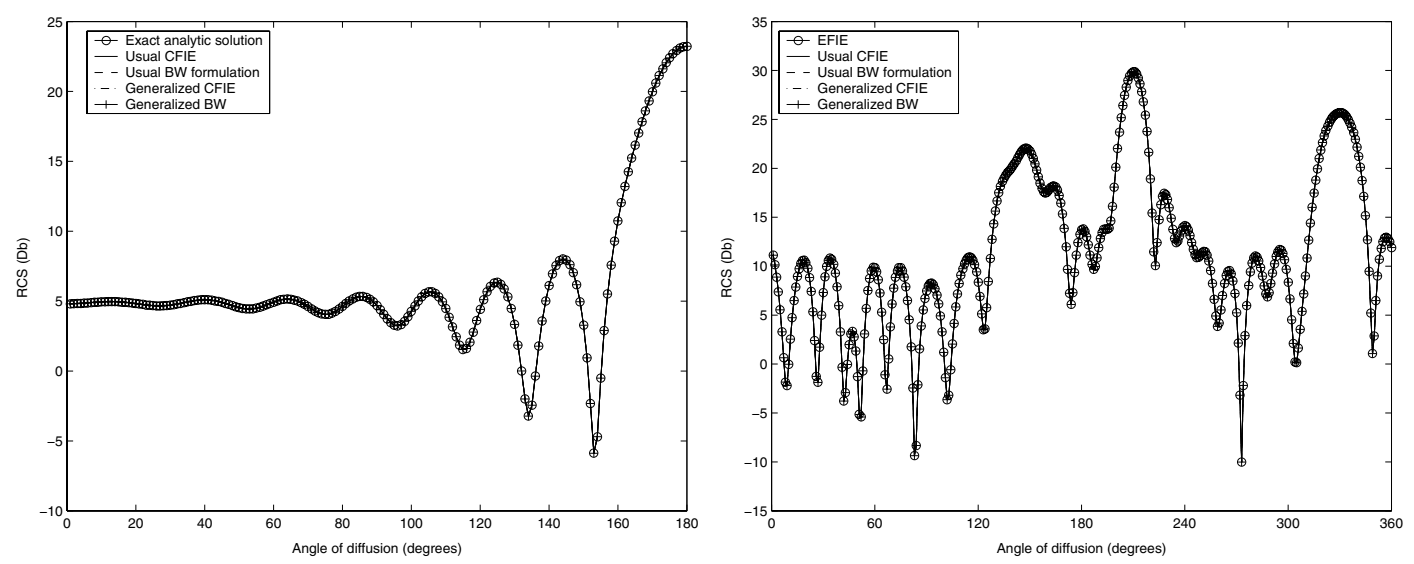

FIGURE 7. Sound-hard problem: accuracy of the representation of the RCS for the unit sphere with $k=13, n_{\lambda}=10$ and $\left(\phi^{\text {inc }}, \theta^{\text {inc }}\right)=(0,0)$ degrees incidence (left figure) and for the cube with $k=10, n_{\lambda}=10$ and $\left(\phi^{\text {inc }}, \theta^{\text {inc }}\right)=(0,30)$ degrees incidence (right figure).

TABLE 1. Unit sphere, Neuman problem for $k=8$ and an incidence of $\left(\phi^{\text {inc }}, \theta^{\text {inc }}\right)=(0,0)$ degrees. The tolerance is fixed to $10^{-8}$ in the GMRES for the resolution of the auxiliary linear system. (nnz: Non-zero elements in a sparse matrix.)

\begin{tabular}{|c|c|c|c|c|}
\hline$N_{V}$ & $n_{\lambda}$ & CPU (sec.) for a dense MVP & CPU time (sec.) for the iterative solution of one sparse system & $\mathrm{nnz}$ \\
\hline 2892 & 10.1 & 0.59 & $2.256 \mathrm{e}-2$ & 20232 \\
\hline 4842 & 13 & 2.98 & $5.375 \mathrm{e}-2$ & 33882 \\
\hline 6762 & 15.4 & 7.61 & $8.875 \mathrm{e}-2$ & 47322 \\
\hline 10892 & 19.6 & 17.57 & 0.164 & 58861 \\
\hline
\end{tabular}

TABLE 2. Unit cube, Neuman problem for $k=8$ and an incidence of $\left(\phi^{\text {inc }}, \theta^{\text {inc }}\right)=(0,30)$ degrees. The tolerance is fixed to $10^{-8}$ in the GMRES for the resolution of the auxiliary linear system. (nnz: Non-zero elements in a sparse matrix.)

\begin{tabular}{|c|c|c|c|c|}
\hline$N_{V}$ & $n_{\lambda}$ & CPU (sec.) for a dense MVP & CPU time (sec.) for the iterative solution of one sparse system & $\mathrm{nnz}$ \\
\hline 3066 & 7 & 0.56 & $2.875 \mathrm{e}-2$ & 19742 \\
\hline 5820 & 9.2 & 4.02 & $7.125 \mathrm{e}-2$ & 38348 \\
\hline 6774 & 9.8 & 6.32 & $8.375 \mathrm{e}-2$ & 44858 \\
\hline 10620 & 12.5 & 15.6 & 0.13 & 76232 \\
\hline
\end{tabular}

wave strikes into the cavity. Therefore, multiple scattering effects arise. We see in Figure 8 that the number of iterations remains independent of $n_{\lambda}$. We also observe that the total number of iterations increases weakly according to $k$ even if the generalized formulations yield a better convergence rate than the classical formulations. This deterioration is a future prospecting and not trivial aspect to improve in the generalized formulations.

\section{Conclusion}

We have proposed in this paper some alternative well-posed integral equations for the acoustic scattering problem by a smooth closed surface. These formulations which generalize the usual Brakhage-Werner and CFIE integral equations have an improved convergence rate when they are solved by a Krylov subspace iterative solver. Their convergence rate is independent of the density of discretization points per wavelength and almost of the 

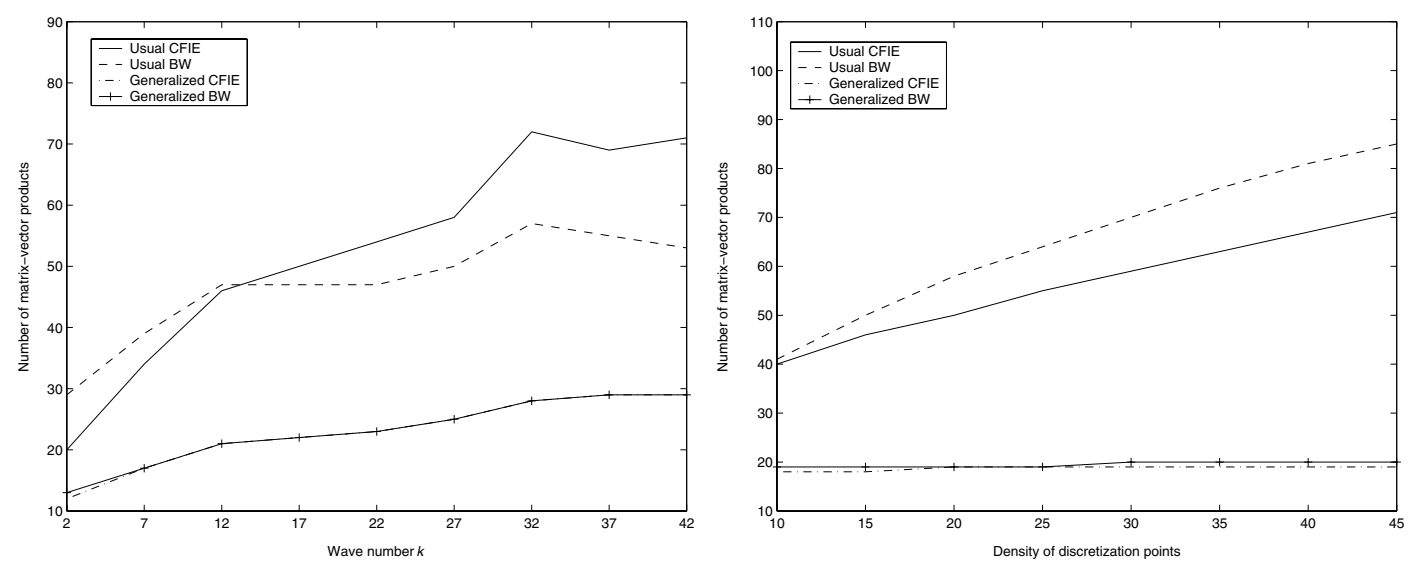

FIGURE 8. Sound-hard square cylinder with cavity: number of matrix-vector products for the four integral formulations with respect to the wavenumber $k$ (left: $n_{\lambda}=10$ and 30 degrees incidence) and the density $n_{\lambda}$ of discretization points per wavelength (right: $k=10$ and 30 degrees incidence).

frequency. These new formulations should be efficient when they are coupled to a fast solver like for instance the FMM $[26,48]$ or the high-order solvers developed in $[12,13]$.

The extension to more general scattering problems is actually under progress (generalized impedance boundary conditions for instance). Moreover, more numerical experiments remain to do for higher frequencies and more complicate shapes to confirm the efficiency of the alternative formulations. The first developments concerning the extension of the generalized equations to the full system of Maxwell's equations are available in [31,32]. The efficiency of the approach is shown in the particular case of a spherical geometry using a vector spherical harmonics decomposition. The numerical approximation of these generalized formulations is under progress. Finally, a comparison with others CFIEs constructed in electromagnetism as for instance in [1] would be an interesting study to conduct.

\section{REFERENCES}

[1] F. Alouges, S. Borel and D. Levadoux, A new well-conditionned integral formulation for Maxwell Equations in three dimensions. IEEE Trans. Ant. Prop. 53 (2005) 2995-3004.

[2] S. Amini and S.M. Kirkup, Solution of Helmholtz equation in exterior domain by elementary boundary integral equations. $J$. Comput. Phys. 118 (1995) 208-221.

[3] S. Amini and N.D. Maines, Preconditioned Krylov subspace methods for boundary element solution of the Helmholtz equation. Internat. J. Numer. Methods Engrg. 41 (1998) 875-898.

[4] X. Antoine, Fast approximate computation of a time-harmonic scattered field using the On-Surface Radiation Condition method. IMA J. Appl. Math. 66 (2001) 83-110.

[5] X. Antoine, Some Applications of the On-Surface Radiation Condition to the Integral Equations for Solving Electromagnetic Scattering Problems. Industrial Mathematics and Statistics, Narosa Publishing (2003).

[6] X. Antoine and M. Darbas, Alternative integral equations for the iterative solution of acoustic scattering problems. Quaterly J. Mech. Appl. Math. 58 (2005) 107-128.

[7] X. Antoine, H. Barucq and A. Bendali, Bayliss-Turkel-like radiation condition on surfaces of arbitrary shape. J. Math. Anal. Appl. 229 (1999) 184-211.

[8] X. Antoine, A. Bendali and M. Darbas, Analytic preconditioners for the electric field integral equation. Internat. J. Numer. Methods Engrg. 61 (2004) 1310-1331.

[9] X. Antoine, M.Darbas and Y.Y. Lu, An improved surface radiation condition for high-frequency acoustics scattering problems. Comput. Meth. Appl. Mech. Eng. 195 (2006) 4060-4074.

[10] J.J. Bowman, T.B.A. Senior and P.L.E. Uslenghi, Electromagnetic and acoustic scattering by simple shapes. North-Holland Publishing Compagny, Amsterdam (1969). 
[11] A. Brakhage and P. Werner, Über das Dirichletsche Aussenraumproblem für die Helmholtzsche Schwingungsgleichung. Arch. Math. 16 (1965) 325-329.

[12] O.P. Bruno and L.A. Kunyansky, A fast, high-order algorithm for the solution of surface scattering problems: basic implementation, tests, and applications. J. Comput. Phys. 169 (2001) 80-110.

[13] O.P. Bruno and L.A. Kunyansky, Surface scattering in three dimensions: an accelerated high-order solver. P. R. Soc. Lond. Proc. Ser. A Math. Phys. Eng. Sci. 457 (2001) 2921-2934.

[14] A. Buffa and R. Hiptmair, A coercive combined field integral equation for electromagnetic scattering. SIAM J. Numer. Anal. 42 (2004) 621-640.

[15] A. Buffa and R. Hiptmair, Regularized combined field integral equations. Numer. Math. 100 (2005) 1-19.

[16] D.C. Calvo, M.D. Collins and D.K. Dacol, A higher-order on-surface radiation condition derived from an analytic representation of a Dirichlet-to-Neumann map. IEEE. Trans. Antennas Progat. 51 (2003) 1607-1614.

[17] S.L. Campbell, I.C.F. Ipsen, C.T. Kelley, C.D. Meyer and Z.Q. Xue, Convergence estimates for solution of integral equations with GMRES. J. Integral Equations Appl. 8 (1996) 19-34.

[18] B. Carpintieri, I.S. Duff and L. Giraud, Experiments with sparse approximate preconditioning of dense linear problems from electromagnetic applications. Technical Report TR/PA/00/04, Cerfacs, France (2000).

[19] B. Carpintieri, I.S. Duff and L. Giraud, Sparse pattern selection strategies for robust Froebenius norm minimization preconditioners in electromagnetism, Preconditioning Techniques for Large Sparse Matrix Problems in Industrial Applications (Minneapolis, MN, 1999). Numer. Lin. Alg. Appl. 7 (2000) 667-685.

[20] G. Chen and J. Zhou, Boundary Element Methods. Academic Press, Harcourt Brace Jovanovitch, Publishers (1992).

[21] K. Chen, On a class of preconditioning methods for dense linear systems from boundary elements. SIAM J. Sci. Comput. 20 (1998) 684-698.

[22] K. Chen, Discrete wavelet transforms accelerated sparse preconditioners for dense boundary element systems. Electron. Trans. Numer. Anal. 8 (1999) 138-153.

[23] K. Chen, An analysis of sparse approximate inverse preconditioners for boundary elements. SIAM J. Matrix Anal. Appl. 22 (2001) 1958-1978.

[24] K. Chen and P.J. Harris, Efficient preconditioners for iterative solution of the boundary element equations for the threedimensional Helmholtz equation. Appl. Numer. Math. 36 (2001) 475-489.

[25] W.C. Chew and Warnick, On the spectrum of the electric field integral equation and the convergence of the moment method. Internat. J. Numer. Methods Engrg. 51 (2001) 31-56.

[26] W.C. Chew, J-M. Jin, E. Michielssen and J. Song, Fast and Efficient Algorithms in Computational Electromagnetics. Artech House Antennas and Propagation Library, Norwood (2001).

[27] S.H. Christiansen and J.C. Nédélec, Des préconditionneurs pour la résolution numérique des équations intégrales de frontière de l'acoustique. C.R. Acad. Sci. Paris, Sér. I 330 (2000) 617-622.

[28] S.H. Christiansen and J.C. Nédélec, A preconditioner for the electric field integral equation based on Calderon formulas. SIAM J. Numer. Anal. 40 (2002) 1100-1135.

[29] D. Colton and R. Kress, Integral Equations in Scattering Theory. Pure and Applied Mathematics, John Wiley and Sons, New York (1983).

[30] D. Colton and R. Kress, Inverse Acoustic and Electromagnetic Scattering Theory. Second Edition, Applied Mathematical Sciences 93, Springer-Verlag, Berlin (1998).

[31] M. Darbas, Préconditionneurs Analytiques de type Calderòn pour les Formulations Intégrales des Problèmes de Diffraction d'ondes. Ph.D. Thesis, Université P. Sabatier, Toulouse, France (November 2004).

[32] M. Darbas, Generalized CFIE for the iterative solution of 3-D Maxwell Equations. Appl. Math. Lett. 19 (2006) 834-839.

[33] J.M. Ford, An improved discrete wavelet transform preconditioner for dense matrix problems. SIAM J. Matrix Anal. Appl. 25 (2003) 642-661.

[34] R.F. Harrington and J.R. Mautz, H-field, E-field and combined field solution for conducting bodies of revolution. Arch. Elektron. Übertragungstech (AË̈) 32 (1978) 157-164.

[35] P.L. Ho and Y.Y. Lu, Improving the beam propagation method for TM polarization. Opt. Quant. Electron. 35 (2003) $507-519$.

[36] D.S. Jones, Surface radiation conditions. IMA J. Appl. Math. 41 (1988) 21-30.

[37] D.S. Jones, An approximate boundary condition in acoustics. J. Sound Vibr. 121 (1988) 37-45.

[38] D.S. Jones, An improved surface radiation condition. IMA J. Appl. Math. 48 (1992) 163-193.

[39] C.T. Kelley and Z.Q. Xue, GMRES and integral operators. SIAM J. Sci. Comput. 17 (1996) 217-226.

[40] R. Kress, Minimizing the condition number of boundary integral operators in acoustic and electromagnetic scattering. Quaterly J. Mech. Appl. Math. 38 (1985) 323-341.

[41] G.A. Kriegsmann, A. Taflove and K.R. Umashankar, A new formulation of electromagnetic wave scattering using the on-surface radiation condition method. IEEE Trans. Antennas Propag. 35 (1987) 153-161.

[42] D.P. Levadoux and B.L. Michielsen, Analysis of a boundary integral equation for high frequency Helmholtz equation, 4th International Conference on Mathematical and Numerical Aspects of Wave Propagation, Golden, Colorado, 1-5 June (1998) $765-767$. 
[43] D.L. Levadoux and B.L. Michielsen, New integral equation formulations for wave scattering problems. ESAIM: M2AN 38 (2004) $157-176$.

[44] Y.Y. Lu and P.L. Ho, Beam propagation method using a $[(p-1) / p]$ Padé approximant of the propagator. Opt. Lett. 27 (2002) 683-685.

[45] W. Mc Lean, Strongly Elliptic Systems and Boundary Integral Equations. Cambridge University Press, Cambridge, UK (2000).

[46] F.A. Milinazzo, C.A. Zala, G.H. Brooke, Rational square-root approximations for parabolic equation algorithms. J. Acoust. Soc. Am. 101 (1997) 760-766

[47] I. Moret, A note on the superlinear convergence of GMRES. SIAM J. Numer. Anal. 34 (1997) 513-516.

[48] V. Rokhlin, Rapid solution of integral equations of scattering theory in two dimensions. J. Comput. Phys. 86 (1990) $414-439$.

[49] Y. Saad, Iterative Methods for Sparse Linear Systems. PWS Pub. Co., Boston (1996).

[50] Y. Saad and M.H. Schultz, GMRES: a generalized minimal residual algorithm for solving nonsymmetric linear systems. SIAM J. Sci. Statist. Comput. 7 (1986) 856-869.

[51] O. Steinbach and W.L. Wendland, The construction of some efficient preconditioners in the boundary element method. Adv. Comput. Math. 9 (1998) 191-216.

[52] D. Yevick, A guide to electric-field propagation techniques for guided-wave optics. Opt. Quant. Electron. 26 (1994) $185-197$. 